\title{
Fear conditioned changes of heart rate in patients with medial cerebellar lesions
}

\author{
M Maschke, M Schugens, K Kindsvater, J Drepper, F P Kolb, H-C Diener, I Daum, \\ D Timmann
}

Fear conditioned changes of heart rate and skin conductance responses were investigated in patients with medial cerebellar lesions. A classical conditioning paradigm with a tone as the conditioned stimulus (CS) and an electrical shock as the unconditioned stimulus (US) was tested on five patients with medial cerebellar lesions due to surgery for astrocytoma and five controls. The CS preceded the US by $5900 \mathrm{~ms}$ and coterminated with the US. Changes in heart rate and skin conductance responses were obtained as measures for autonomic fear responses. Effects of conditioning were quantified by comparison of the habituation and extinction phases. Controls, but not cerebellar patients, showed a significant decrease of heart rate during fear conditioning. However, there were no significant fear conditioned changes in electrodermal responses in either group. In summary, the medial cerebellum seems to be involved in fear-conditioned bradycardia in humans.

t

nvolvement of the cerebellum is well recognised in associative and non-associative motor learning of different specific and non-specific aversive reactions in various species. ${ }^{12}$ In humans, the intermediate cerebellum is known to take part in classical conditioning of specific aversive reactions-that is, conditioning of the eyeblink, ${ }^{3}$ limb flexion, ${ }^{4}$ and jaw opening reflexes. ${ }^{5}$ More recent studies suggest a role of the medial cerebellum in fear conditioned potentiation of the acoustic blink reflex $^{6}$ and long term habituation of the acoustic startle response. ${ }^{7}$ Impaired conditioning of another non-specific aversive reaction, fear conditioned bradycardia, has been found in animal lesion studies of the medial cerebellum..$^{8-11}$ The aim of the present study was to examine whether the cerebellum takes part in fear conditioning in humans, assessed as changes in heart rate and skin conductance responses.

\section{PATIENTS AND METHODS}

Five patients with cerebellar lesions (four males, one female, mean age 19.6 (SD 5.2) years) and five age and sex matched healthy controls (four males, one female, mean age 21.2 (SD 5.8) years) participated. All patients had medial cerebellar lesions-including the vermis-due to surgery for midline cerebellar astrocytoma. The severity of ataxia was scored using the international cooperative ataxia rating score of the World Federation of Neurology (WFN score). ${ }^{12}$ Brain MRI and clinical examination excluded extracerebellar lesions. Descriptive statistics of the patients and precise localisation of the cerebellar lesion as evaluated on MR images are shown in table 1. All patients and healthy subjects gave informed consent. The ethics committee of the Medical School, University of Essen, approved the project.

For testing of fear conditioning, a standard delay paradigm was performed in which a tone (conditioned stimulus (CS)) coterminated with an electrical shock (unconditioned stimulus (US)). The paradigm consisted of three phases: (1) habituation phase (six CS alone trials), (2) conditioning phase (20 pairs of CS and US), and, (3) extinction phase (six CS alone trials). The mean intertrial interval was 25 seconds (range $22-28 \mathrm{~s})$. The tone $(70 \mathrm{~dB}, 1000 \mathrm{~Hz})$ was presented bilaterally via stereophonic headphones. During intertrial intervals subjects received white noise $(50 \mathrm{~dB})$ to mask environmental noise. The tone (CS) lasted for 6 seconds and preceded the US by $5900 \mathrm{~ms}$. The electric shock (US, duration $100 \mathrm{~ms}, 100 \mathrm{~Hz}$, $0.1 \mathrm{~ms}$ ) was applied to the subject's right index finger via a bipolar stimulus electrode with the individually highest tolerable intensity (patients: mean 4.5 (SD 3.2) mA, range 1.2-9.5 $\mathrm{mA}$; controls: mean 5.4 (SD 1.0) $\mathrm{mA}$, range $4.8-6.8, t$ test $\mathrm{p}>0.5)$. After the end of the experiment, subjects were asked to name a number from 0 to 10 to describe their subjective intensity of pain during the experiment with 0 representing "no pain" and 10 the "strongest pain you can imagine". Pain ratings were not significantly different between patients and control subjects (patients: mean 7.3 (SD 1.9), range 4.0-8.5; controls: mean 5.4 (SD 2.2), range 3.5-9.0, $t$ test $\mathrm{p}=0.2$ ).

Heart rate and skin conductance responses were obtained from 6 seconds before CS onset until 6 seconds after CS offset (total analysis time $18 \mathrm{~s}$ ). Heart rate was recorded via limb leads. Heart rate recordings were filtered off line (20 $\mathrm{Hz} \leqslant \mathrm{f} \leqslant 200 \mathrm{~Hz}$ ). For each beat to beat interval corresponding beats per minute (bpm) were calculated. Skin conductance was obtained using two electrodes placed at the ball of the thumb and the fifth finger of the non-dominant hand. Incidence of both the first and second interval responses (FIR and SIR) were measured using standard criteria defined by Boucsein. ${ }^{13}$ The respiratory rate was obtained by a thermistor placed near to one nostril to control for respiratory related changes in heart rate. Respiratory rate was not significantly different between the groups throughout the experiment $(t$ test $\mathrm{p}>0.2$ ).

According to other conditioning studies, conditioning effects of changes of heart rate and skin conductance responses were expected to be most prominent in the interval from 4 to 6 seconds after onset of the CS (second half of the CS-US interval in the conditioning phase). ${ }^{14}{ }^{15}$ Therefore, we analysed the SIR and changes of heart rate within this interval. To allow intersubject and group comparisons, in each subject the mean heart rate in the 2 scond interval before onset of the CS was subtracted from the mean heart rate in the interval from 4 to 6 seconds after CS onset (meandiff/bpm). The SIR incidence was used to assess electrodermal conditioning.

Abbreviations: CS, conditioned stimulus; US, unconditioned stimulus; WFN score, international cooperative ataxia rating score of the World Federation of Neurology; FIR, first interval response; SIR, second interval response 
Table 1 Basic characteristics of patients with medial cerebellar lesions due to surgery for astrocytoma

\begin{tabular}{|c|c|c|c|c|c|}
\hline Patient & Age (y) & Sex & $\begin{array}{l}\text { Date of } \\
\text { operation }\end{array}$ & $\begin{array}{l}\text { Localisation of vermal } \\
\text { lesions (Larsell lobules) }\end{array}$ & $\begin{array}{l}\text { Total WFN } \\
\text { ataxia score }\end{array}$ \\
\hline 1 & 21 & $M$ & 1994 & VI-VIII, (IX) & $8 / 100$ \\
\hline 2 & 23 & $M$ & 1995 & IV-VII, (III, VIII) & $5 / 100$ \\
\hline 3 & 25 & $M$ & 1992 & VI-VIII, (IV, V, IX, X) & $6 / 100$ \\
\hline 4 & 12 & $\mathrm{~F}$ & 1996 & VII, VIII, (VI, IX, X) & $21 / 100$ \\
\hline 5 & 17 & $M$ & 1992 & VI, VII, (II-V) & $28 / 100$ \\
\hline
\end{tabular}

For data reduction, the means of meandiff/bpm and SIR incidence of five successive trials were calculated (trial 3-7=block 1 (habituation phase), trial $8-27=$ block $2-5$ (conditioning phase), trial $28-32=$ block 6 (extinction phase)). Effects of fear conditioning were quantified by comparing results of autonomic response parameters of the habituation phase with those of the extinction phase (block $1 v$ block 6). ${ }^{16}$

\section{RESULTS}

Univariate repeated measures analyses of variance (ANOVA) with meandiff/bpm and SIR incidence as dependent variables, group (controls $v$ cerebellar patients) as between subjects factor, and block (habituation $v$ extinction) as within subject factor were performed. Changes in heart rate showed no significant difference between groups $(F=0.54 ; \mathrm{p}=0.48)$ or blocks $(F=2.71 ; \mathrm{p}=0.14)$. The group by block interaction almost reached significance $(F=4.81 ; \mathrm{p}=0.06)$ indicating differences in heart rate conditioning between groups. In controls, inspection of mean values showed a decrease of heart rate in the interval from 4 to 6 seconds after CS onset in the extinction phase compared with the habituation phase (fig l A) (mean (SD) meandiff/bpm 2.4 (5.5) (block 1) $v 0.1$ (3.7) (block 6)). In cerebellar patients, however, the heart rate increased in the extinction phase compared with the habituation phase (mean (SD) meandiff/bpm -1.8 (5.7) (block 1) $v$ 0.6 (3.4) (block 6)).

To control intersubject variability of baseline heart rate, the errors of the predicted heart rate (residuals) within the interval from 4 to 6 seconds after CS onset were calculated. For the calculation of the residuals, a linear regression analysis with heart rate of $2 \mathrm{~s}$ interval before onset of the CS as predictor variable and heart rate of the interval from 4 to 6 seconds after CS onset as dependent variable in each subject was performed. Mean residuals were calculated by subtracting the predicted heart rate from the obtained heart rate within the interval from 4 to 6 seconds after CS onset. Again, univariate repeated measures analysis with mean residuals of heart rate as dependent variable showed no significant group or block effect $(F=1.29 ; \mathrm{p}=0.3)$. However, there was a significant group by block interaction effect $(F=11.78 ; \mathrm{p}=0,009)$ (fig $1 \mathrm{~B})$, which confirmed differences in heart rate conditioning between groups as shown for meandiff/bpm change (fig l A).

Finally, changes in heart rate were compared between both groups within the habituation phase to exclude possible differences in resting heart rate and habituation of the orienting response. Univariate repeated measures analyses with meandiff/bpm and residuals of heart rate in the interval from 4 to 6 seconds after CS onset of the habituation phase as dependent variables, group as between subject factor and trial (1-7) as within subject factor showed no significant group, trial, or group by trial effect (all p values $>0.2$ ).

The frequency of SIRs was higher in the extinction phase compared with the habituation phase both in cerebellar patients and controls (mean frequency SIR (SEM); controls $0.17(0.06)$ (block 1) v $0.28(0.09)$ (block 6); cerebellar patients 0.22 (0.07) (block l) $v 0.24$ (0.09) (block 6)) (fig l C). However, the univariate repeated measures analysis showed no significant conditioning effects (group, block, and group by block interaction effects: all p values $>0.2$ ).

\section{DISCUSSION}

Our findings of missing fear conditioned heart rate changes in cerebellar patients agree with animal lesion studies, ${ }^{10}{ }^{11}$ which showed impaired acquisition of conditioned bradycardic responses after removal of the cerebellar vermis. Moreover, Lavond $e t a l^{9}$ found no change in heart rate conditioning in animals with lesions of the dentate and interpositus cerebellar nuclei, which further suggests a selective involvement of the medial cerebellum in conditioning of the heart rate.

It may be argued that abnormal resting heart rate or a general inability to change heart rate accounts for deficits in fear conditioned bradycardia in cerebellar patients. The present study only studied the heart response to the CS during the habituation phase as a parameter for resting heart rate and general responsiveness to stimuli-that is, orienting response, and did not find differences between cerebellar patients and controls. This is supported by previous animal lesion studies, ${ }^{11}$ which reported no changes in the orienting response
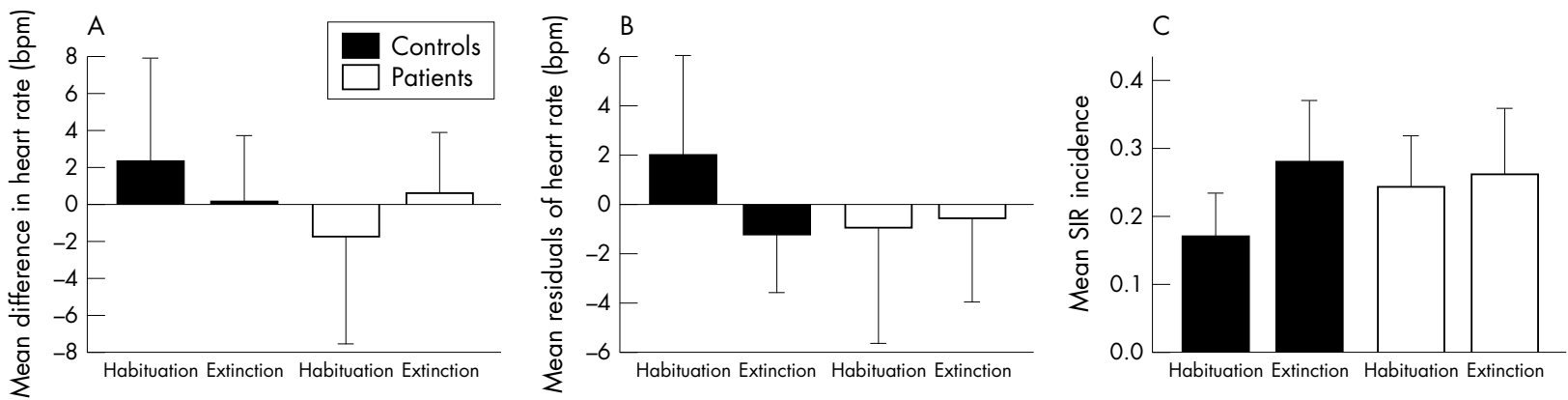

Figure 1 Fear conditioned bradycardia and electrodermal responses. Means (SD) of (A) differences in heart rate (meandiff/bpm, (B) residuals of heart rate, and (C) means (SEM) of the second interval response (SIR) incidence of the electrodermal response in the habituation and extinction phases in cerebellar patients and the control group. Note the decrease in the difference in heart rate as well as in residuals in the extinction phase compared with the habituation phase in the control group, but not in cerebellar patients. Mean SIR incidence increased in the extinction phase in both groups, but the difference did not reach significance. 
after ablation of the cerebellar vermis. However, the fastigial nucleus has been suggested as being involved in autonomic and vasomotor regulation ${ }^{17}$ and a recent PET study provided evidence for an involvement of the human vermal cerebellum in autonomic cardiovascular control. ${ }^{18}$ Thus, from the present data the possibility cannot be excluded that a more general inability to change autonomic responses is at least in part responsible for the missing fear conditioned heart rate changes in cerebellar patients. Further studies are needed to quantify the cerebellar involvement in unconditioned heart rate changes with US alone trials using fearful and non-threatening stimuli and other tests such as isometric exercise and heart rate changes to inspiration/expiration.

By contrast with fear conditioned bradycardia in healthy controls, no significant effects of fear conditioning of electrodermal responses were present in the control (and cerebellar) group. In both groups, however, there was a tendency of the SIR incidence to increase in the extinction phase compared with the habituation phase. Effects of electrodermal fear conditioning may be less prominent due to the few subjects tested and the known high intersubject variability of skin conductance responses. ${ }^{14}$ Moreover, other human studies failed to show differences in fear conditioned electrodermal responses comparing control patients and and cerebellar subjects. ${ }^{6} 19$ However, given the long electrodermal response onsets (up to $3 \mathrm{~s}$ ) results of these studies have to be interpreted with caution because they used a shorter CS-US interval $(<1 \mathrm{~s}, 2 \mathrm{~s})$ than in the present study $(6 \mathrm{~s})$. Thus, to decide whether or not the cerebellum is involved in electrodermal fear conditioning a study needs to be performed including a larger number of patients and controls and using an optimal long CS-US interval.

In summary, the present findings suggest a role for the human medial cerebellum in fear conditioned bradycardia. Recent studies of our group showed an involvement of the medial cerebellum in fear conditioned potentiation of the acoustic blink reflex ${ }^{6}$ and long term habituation of the acoustic startle response in humans. ${ }^{720}$ Thus, the medial cerebellum seems to be involved both in non-associative and associative learning of various non-specific aversive reactions. Furthermore, the present data are consistent with an increasingly recognised role of the cerebellum in emotion and cognition. ${ }^{21}$ Further studies with larger study populations are needed to identify the specific role of the medial cerebellum in the regulation of autonomic responses and to confirm data of the present study.

\section{Authors' affiliations}

M Maschke, K Kindsvater, J Drepper, H-C Diener, D Timmann, Department of Neurology, University of Essen, Hufelandstrasse 55 45122 Essen, Germany

M Schugens, I Daum, Department of Psychology, University of Bochum, GAFO 05/612, 44780 Bochum, Germany
F P Kolb, Institute of Physiology, University of Munich, Pettenkoferstrasse 12, 80336 Munich, Germany

Correspondence to: Dr M Maschke, Department of Neurology, University of Essen, Hufelandstrasse 55, 45122 Essen, Germany;

matthias.maschke@uni-essen.de

Received 8 March 200

In final revised form 21 August 2001

Accepted 30 August 2001

\section{REFERENCES}

1 Bloedel JR, Bracha V. On the cerebellum, cutaneomuscular reflexes, movement control and elusive engrams of memory. Behav Brain Res 1995;68: 1-44.

2 Thompson RF, Krupa DJ. Organization of memory traces in the mammalian brain. Annu Rev Neurosci 1994;17:519-49.

3 Woodruff-Pak DS. Classical conditioning. In: Schmahmann JD, ed. The cerebellum and cognition. International review of neurobiology. Vol 41. San Diego: American Press, 1997:342-66.

4 Timmann D, Baier PC, Diener HC, et al. Classically conditioned withdrawal responses in cerebellar patients. 1. Impaired conditioned responses. Exp Brain Res 2000;130:453-70.

5 Maschke M, Kolb FP, Drepper J, et al. A possible role of the human cerebellum in conditioning of the jaw-opening reflex. Neurosci Lett 2000a:285:213-17.

6 Maschke M, Drepper J, Kindsvater K, et al. Fear conditioned potentiation of the acoustic blink reflex in patients with cerebellar lesions. J Neurol Neurosurg Psychiatry 2000;68:358-64.

7 Maschke M, Drepper J, Kindsvater K, et al. Involvement of the medial cerebellum in long-term habituation of the acoustic startle response. Exp Brain Res 2000c;133:359-67.

8 Ghelarducci B, Salamone D, Simoni A, et al. Effects of early cerebellar removal in the classically conditioned bradycardia of adult rabbits. Exp Brain Res 1996;111:417-23.

9 Lavond DG, Lincoln JS, McCormick DA, et al. Effect of bilateral lesions of the dentate and interpositus cerebellar nuclei on conditioning of heart-rate and nictitating membrane/eyelid responses in the rabbit. Brain Res 1984;305:323-30.

10 Sebastiani L, LaNoce A, Paton JF, et al. Influence of the cerebellar posterior vermis on the acquisition of the classically conditioned bradycardia response in the rabbit. Exp Brain Res 1992;88:193-8.

11 Supple WF, Leaton RN. Cerebellar vermis: essential for classically conditioned bradycardia in the rat. Brain Res 1990;409:17-23.

12 Trouillas $\mathbf{P}$, Takayangi T, Hallett $M$, et al. International cooperative ataxia rating score for pharmacological assessment of the cerebella syndrome. J Neurol Sci 1997;45:205-11.

13 Boucsein W. Electrodermal activity. New York: Plenum Press, 1992

14 Öhmann A. Orienting reactions, expectancy learning, and conditional responses in electrodermal conditioning with different interstimulus intervals. Biol Psychol 1974;1:189-200.

15 Powell DA, Lipkin M, Milligan ML. Concomitant heart rate and corneoretinal potential conditioning in the rabbit (Oryctolagus cuniculus): effects of caudate lesions. Learning and Motivation 1974;5:532-47.

16 Büchel C, Dolan RJ. Classical fear conditioning in functional neuroimaging. Curr Opin Neurol 2000;10:219-23.

17 Reis DJ, Golanov EV. Autonomic and vasomotor regulation. In: Schmahmann JD, ed. The cerebellum and cognition. International review of neurobiology. Vol 41. San Diego: American Press, 1997:121-49.

18 Critcheley HD, Corfield DR, Chandler MP, et al. Cerebral correlates of autonomic cardiovascular arousal: a functional neuroimaging investigation in humans. J Physiol 2000,523:259-70.

19 Daum I, Schugens MM, Ackermann H, et al. Classical conditioning afte cerebellar lesions in humans. Behav Neurosci 1993;107:748-56.

20 Timmann D, Musso C, Kolb FP, et al. Involvement of the human cerebellum during habituation of the acoustic startle response: a PET study. J Neurol Neurosurg Psychiatry 1998;65:771-3.

21 Schmahmann JD, Sherman JC. Cerebellar cognitive affective syndrome. Brain 1998;121:561-79. 